\title{
Production of a Cloned Offspring and CRISPR/Cas9 Genome Editing of Embryonic Fibroblasts in Cattle
}

\author{
G. N. Singina ${ }^{a, *}$, P. V. Sergiev ${ }^{b, c, d}$, A. V. Lopukhov ${ }^{a}$, M. P. Rubtsova ${ }^{d}$, N. P. Taradajnic ${ }^{a}$, N. V. Ravin ${ }^{e}$, \\ E. N. Shedova ${ }^{a}$, T. E. Taradajnic ${ }^{a}$, I. A. Polejaeva ${ }^{f}$, A. V. Dozev ${ }^{a}$, Foreign Member of the RAS G. Brem ${ }^{g}$, \\ Academician O. A. Dontsova ${ }^{c, d, h, i}$, and Academician N. A. Zinovieva ${ }^{a}$ \\ Received October 10, 2020; revised October 20, 2020; accepted October 21, 2020
}

\begin{abstract}
Somatic Cell Nuclear Transfer (SCNT) technique was used to produce the first viable cloned cattle offspring in Russia. Whole-genome SNP genotyping confirmed that the cloned calf was identical to the fibroblast cell line that was used for SCNT. CRISPR/Cas9 approach was subsequently used to knock out genes for beta-lactoglobulin gene (PAEP) and the beta-lactoglobulin-like protein gene (LOC100848610) in the fibroblast cells. Gene editing (GE) efficiency was $4.4 \%$ for each of these genes. We successfully obtained singlecell-derived fibroblast colonies containing PAEP and LOC100848610 knockouts, which will be used to produce beta-lactoglobulin-deficient cattle.
\end{abstract}

Keywords: Bos taurus, somatic cloning, gene editing, beta-lactoglobulin knock-out

DOI: $10.1134 /$ S1607672921010099

The gene editing (GE) technology in combination with the technology of somatic cell nuclear transfer (SCNT) has broad prospects for solving problems associated with creating new genotypes, including those with modified economically useful traits [1, 2]. In particular, obtaining cloned embryos using embryonic fibroblasts, in the genome of which the $\beta$-lactoglobulin gene ( $B G L)$ is inactivated, and their transplantation to recipient animals is expected to produce cows capable of producing milk with reduced allergenic properties [3]. The use of a CRISPR/Cas9based system as a tool for editing the genome of

\footnotetext{
${ }^{a}$ Ernst Federal Science Center for Animal Husbandry, Podolsk, Russia

${ }^{b}$ Institute of Functional Genomics, Moscow State University, Moscow, Russia

${ }^{c}$ Center of Life Sciences, Skolkovo Institute of Science and Technology, Skolkovo, Russia

${ }^{d}$ Faculty of Chemistry, Moscow State University, Moscow, Russia

${ }^{e}$ Research Center of Biotechnology, Moscow, Russia

${ }^{f}$ Department of Animal, Dairy and Veterinary Sciences, Utah State University, Logan, UT, USA

${ }^{g}$ Department of Animal Breeding and Genetics, University of Veterinary Medicine, Vienna, Austria

${ }^{h}$ Belozersky Research Institute of Physico-Chemical Biology, Moscow State University, Moscow, Russia

${ }^{i}$ Shemyakin-Ovchinnikov Institute of Bioorganic Chemistry, Moscow, Russia

*e-mail: g_singina@mail.ru
}

somatic cells should be considered as the most effective and innovative approach that is increasingly being used in domestic animals [4, 5]. The SCNT method allows selection of mutant cells prior to the start of expensive animal experiments and ensures obtaining offspring with desired gene modifications. The attractiveness of the CRISPR/Cas9-based system is determined by its high efficiency, simplicity, and low labor intensity [6].

The goal of this study was to construct SCNTembryos of cattle using embryonic fibroblasts as donors of nuclei, to assess their development to viable offspring, as well as to obtain a culture of individual fibroblasts with knocked-out $\beta$-lactoglobulin gene.

For SCNT, post mortem isolated oocyte-cumulus complexes (OCC, $n=1332$ ) were matured in TC-199 medium supplemented with $10 \%$ fetal bovine serum, $10 \mu \mathrm{g} / \mathrm{mL}$ follicle-stimulating hormone (FSH), and $10 \mu \mathrm{g} / \mathrm{mL}$ luteinizing hormones (LH). After 20-24 h of maturation, the OCCs was treated with $0.1 \%$ hyaluronidase solution, cumulus cells were mechanically removed, and oocytes with the first polar body (PPT, $n=1088$ ) were collected. Embryonic fibroblasts were cultured to a formed monolayer, inhibited by contact for 2 days, and prepared in the form of a suspension for subsequent transfer to an enucleated oocyte (Fig. 1a). To fuse oocytes and cells transferred into their perivitelline space, two successive DC pulses with a voltage of $35 \mathrm{~V}$ and a duration of $20 \mu \mathrm{s}$ were used (once or twice in the absence of signs of cell fusion). The resulting cytohybrids $(n=422,44.5 \%$ of the number of oocytes with PPT) were activated with ionomycin $2 \mathrm{~h}$ 

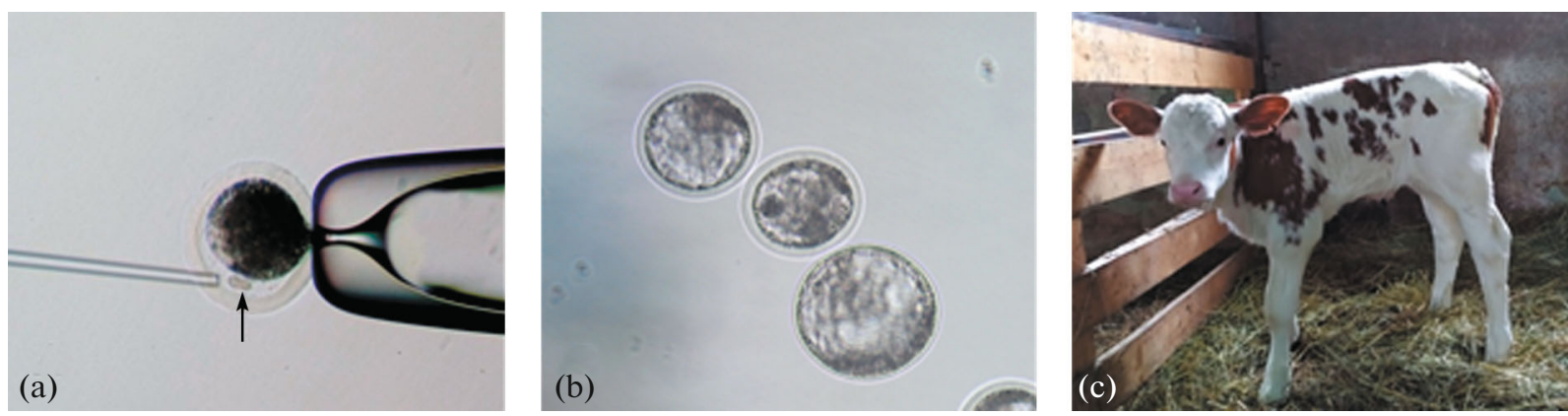

Fig. 1. Photographs of (a) the procedure of somatic cell transfer (shown with the arrow) into the perivitelline space of an enucleated oocyte, (b) cloned bovine embryos used for transplantation to recipient animals, and (c) cloned calf (obtained in Russia for the first time).

(a)

\section{CTGCAGAGCTCAGAAGCGTGACCCCAGCTGCAG CCATGAAGTG CCTCCTG CTTG CCCTGGCCCTCACC TGTGGCG CCCAG GCCCTCATTGTCACCCAGACCATGAAGGG CCTG GATATCCAGAAGGTTCGAGGGT GCCCG}

(b)

\section{ATGAAGTGCTTCCTGCTCGCCCTGGG CCTGGCCCTGGCCTGTGGCATCCAGGCTGCCTACATCCCCCA GATGGCAGGAGACCTGGACATTAGAAAGGTGTGGG GTCTGGG GTGGAGG GTGG GCGCCAGG G GCT GGGGTCCGGGGCTGAGAGG GGACAGGAAAGCCTG GACTGCAGAAGTCTAACGCG GGACCA}

Fig. 2. Scheme of the regions of (a) PAEP (BLG) and (b) LOC100848610 (BLG-like protein) genes in the Bos taurus genome, which were selected as target for gene editing. The hybridization regions of primers used for amplifying genomic fragments are shown in blue. The presumable site of DNA cleavages by the CRISPR/Cas9 system are shown in purple. The hybridization regions of guide RNAs are shown in yellow.

after fusion and cultured to the blastocyst stage (Fig. 1b). To assess the normality and viability of the obtained embryos, some of them $(n=81)$ were transplanted to recipients synchronized in the cycle, and the remaining blastocysts were used for cytological analysis of the state of the nuclear material $(n=16$; average number of nuclei, 78.3). Heifers of breeding age were used as recipients in a spontaneous and synchronized cycle. Before transplantation, sacral epidural anesthesia was performed with a $2 \%$ novocaine solution. Embryo transfer (1-6 embryos per animal) was performed by a non-surgical method deep into the uterine horn.

It was found that, after activation, $64.5 \%$ (272/422) of cytohybrids formed 2-cell embryos and $23.0 \%$ (97/422) develop to the blastocyst stage. The proportion of pregnant animals after transplantation of the cloned blastocysts to 31 recipients was $43.8 \%(14 / 31)$, and the proportion of live births was $3.3 \%(1 / 31)$ (Fig. 1c).

To assess the origin of the obtained calf, genomewide SNP genotyping of the offspring and the cell line was performed using the Bovine GGP HD high-density DNA chip (Neogen/Illumina Inc., United States). To estimate the degree of similarity, the IBS (identity by state) distance values were calculated using the PLINK 1.9 software. IBS distance values for the calve-cell line pairs were 1000 , which confirms the identity of the genotype of the obtained calf and the cell line (for comparison, the IBS distances between unrelated animals of the Simmental breed vary in the range $0.731-0.763)$.

Taking into account the fact that, in the bovine genome, the beta-lactoglobulin $(B L G)$ gene is duplicated and represented by two closely related paralogs, $B L G$ (PAEP) itself and BLG-like gene (LOC100848610), we selected a strategy involving inactivation of both paralogous genes (Fig. 2).

When creating a genetic construct based on CRISPR, the pX458 vector [7] was selected as the basis for inactivation of the PAEP and LOC100848610 genes [7]. This vector contains the hybrid $\operatorname{Cas} 9$ gene and the gene encoding the green fluorescent protein $(G F P)$, the coding regions of which are separated from each other by the sequence encoding the P2A peptide. The pX458 plasmid was cut with the BbsI restriction enzyme. Hybridized oligonucleotides containing guide RNA sequences were ligated from the plasmid backbone. After ligation and transformation of competent $E$. coli JM109 cells, the grown colonies were used to accumulate the biomass and isolate plasmids, 


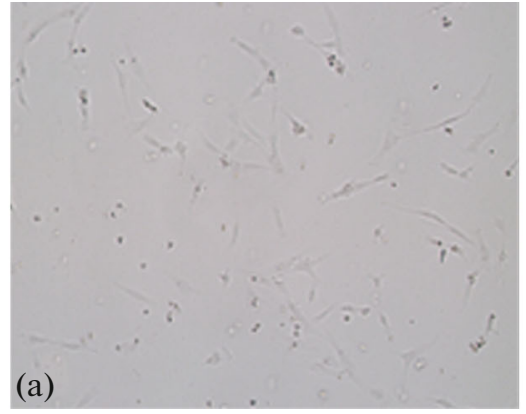

PAEP WT
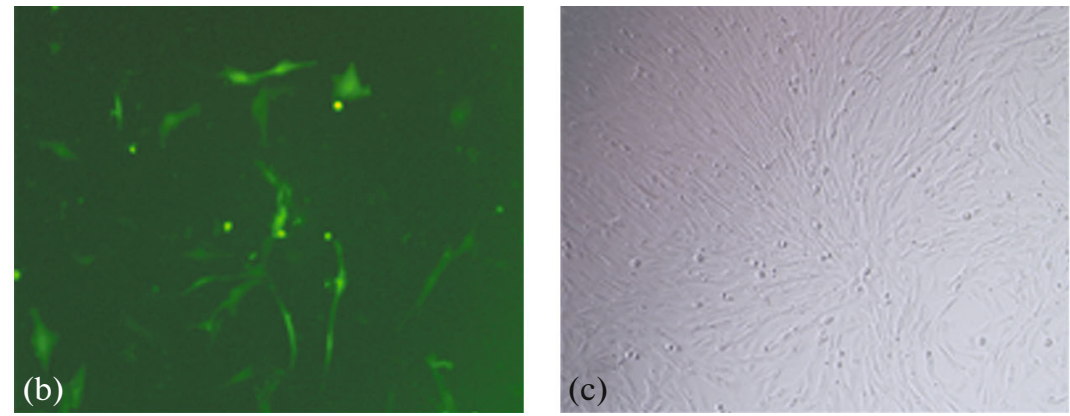

(d)

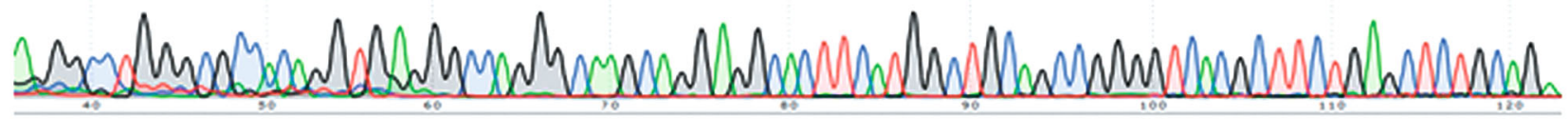

PAEP d $1 /$ WT

A OOCCTOOOCCCCAAOTOAAOOCCCAOOCCAACAAOAAOOAATTCTOOOTOCCACCCOOOTCAAOCC TCCOAACTCCTCCOOA

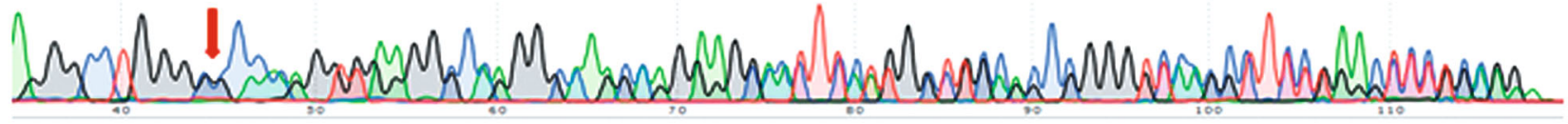

LOC100848610 WT

TOCOTASATCCCCCAOATOOCAOOAOACCTOOACATTAOANAOOTOTOOOOTCTOOOOTOOAOOOTOOOCOCCAOOOOCTOOOOTCCOOOOCTOAOAOOOOACAOAAAA

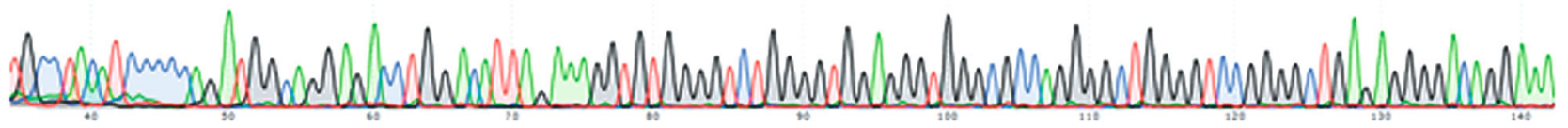

LOC $100848610 \mathrm{~d} 1 / \mathrm{d} 1$

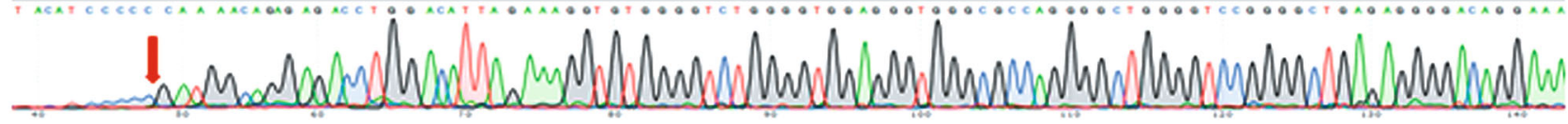

Fig. 3. Micrographs of (a) the total pool of transfected Bos taurus fibroblasts $48 \mathrm{~h}$ after electroporation with a mixture of plasmids encoding Cas9 and gRNA, aimed at inactivating the PAEP and LOC100848610 genes and (b) the cells expressing the genes of the CRISPR/Cas9 and GFP system components (green luminescence). (c) Culture of an individual colony of transfected cells. (d) Results of sequencing the amplicon of the PAEP gene fragment and the LOC100848610 gene. Microinsertions and microdeletions in the region of gene cutting by the CRISPR/Cas9 system can be seen. Genotypes are signed. The sites of mutations are indicated with arrows.

which, on the basis of the confirmation of the successful cloning of the constructs by sequencing, were used for transfection of bovine fibroblasts. The transfected somatic cells carrying the plasmid encoding the components of the CRISPR/Cas9 system were separated from the untransfected cells using the high-throughput BD FACSAria III cell sorter.

High-throughput sequencing of the amplicons of the target regions of the PAEP and LOC100848610 genes in the pre-sorted population of embryonic fibroblasts showed the presence, respectively, of 12 and $7.5 \%$ of mutant sequences containing deletions and insertions of nucleotides at the sites of putative breaks.

To obtain individual colonies of bovine GE fibroblasts, the early-passage fibroblasts were electroporated with a mixture of plasmids encoding Cas9 and gRNA, aimed at inactivating the PAEP and LOC100848610 genes (Fig. 3a).

After sorting (as described above, Fig. 3b), the total pool of the cells expressing the genes for components of the CRISPR/Cas9 system was grown for 2-3 days, after which the cells were seeded individually in 96-well plates and cultured until colonies were obtained (Fig. 3c) and formed $80-90 \%$ of the monolayer. The proportion of the formed colonies was $21.3 \%$ of the total number of cells $(90 / 389)$. One part of each colony was frozen for possible further use, and the other part was used for DNA extraction and analysis for mutations. For this purpose, the PAEP and LOC100848610 gene fragments containing the target regions were amplified, with subsequent Sanger sequencing (Fig. 3d). Knockout of PAEP and LOC100848610 genes was established in 4 out of 90 obtained colonies of individ- 
ual fibroblasts, which corresponds to a gene editing efficiency of $4.4 \%$.

Thus, as a result of the study, the competence of the line of fetal bovine fibroblasts to develop to viable offspring was confirmed by the SCNT method; for the first time, using the CRISPR/Cas9 system, a line of embryonic fibroblasts with knockout of the $P A E P$ and LOC100848610 genes was obtained. The resulting cell line will be used to produce GE offspring of cattle with no beta-lactoglobulin synthesis.

\section{FUNDING}

This work was supported by the Russian Foundation for Basic Research (project no. 18-29-07089) and the Ministry of Science and Higher Education of the Russian Federation.

\section{COMPLIANCE WITH ETHICAL STANDARDS}

Conflict of interest. The authors declare that they have no conflict of interest.

Statement on the welfare of animals. All applicable international, national, and/or institutional guidelines for the care and use of animals were followed.

\section{OPEN ACCESS}

This article is distributed under the terms of the Creative Commons Attribution 4.0 International Public License (http://creativecommons.org/licenses/by/4.0/), which permits unrestricted use, distribution, and reproduction in any medium provided you give appropriate credit to the original author(s) and the source, provide a link to the Creative Commons license, and indicate if changes were made.

\section{REFERENCES}

1. Salamone, D., Barañao, L., Santos, C., et al., High level expression of bioactive recombinant human growth hormone in the milk of a cloned transgenic cow, J. Biotechnol., 2006, vol. 124, no. 2, pp. 469-472.

2. Wang, J., Yang, P., Tang, B., et al., Expression and characterization of bioactive recombinant human alpha-lactalbumin in the milk of transgenic cloned cows, J. Dairy Sci., 2008, vol. 91, no. 12, pp. 4466-4476.

3. Yu, S., Luo, J., Song, Z., et al., Highly efficient modification of beta-lactoglobulin (BLG) gene via zinc-finger nucleases in cattle, Cell Res., 2011, vol. 21, pp. $1638-1640$.

4. Zhou, W., Wan, Y., Guo, R., et al., Generation of betalactoglobulin knockout goats using CRISPR/Cas9, PLoS One, 2017, vol. 12, no. 10. e0186056.

5. Cong, L., Ran, F.A., Cox, D., et al., Multiplex genome engineering using CRISPR/Cas systems, Science, 2013, vol. 339, no. 6121, pp. 819-823.

6. Zinov'eva, N.A., Volkova, N.A., and Bagirov, V.A., Genome editing: state of the art of research and applications in animal husbandry, Biotekhnologiya, 2018, vol. 34, no. 3, pp. 9-22.

7. Ran, F., Hsu, P., Wright, J., et al., Genome engineering using the CRISPR-Cas9 system, Nat. Protocols, 2013, vol. 8, pp. 2281-2308.

Translated by M. Batrukova 